HIV infection, mainly scarifications and/or tattoos (23.1\%), drug abuse (12.9\%) or history of hepatitis B and/or C $(12 \%)$. In cases of unknown sources, PEP was prescribed if the BEA occurred in a suspicious socio-epidemiological context. The PEP was started within four hours of the BEA in $42.8 \%$ of cases and covered 28 days in $56.8 \%$ of cases. Poor compliance with PEP was noted in $55 \%$ of cases. The serological follow-up at one, three and six months was carried out in $11.6 \%, 6.5 \%$ and $4.5 \%$ of cases, respectively. Positive HIV serology of the source was significantly associated with compliance to serological follow-up at one month $(p=0.023)$ and at three months $(p=0.029)$. None of the cases had a seroconversion.

Conclusion A well-managed PEP prevents the risk of HIV seroconversion following a BEA. It is crucial to develop a national updated guideline for management of BEA and PEP.

\section{P-444 SMALL BUSINESS EXPERIENCES WITH COVID-19 PROTECTION MEASURES: A CROSS-SECTIONAL STUDY COMPARING EMPLOYER AND EMPLOYEE PERSPECTIVES IN RURAL NEW BRUNSWICK.}

${ }^{1}$ Kim Cullen, Emily Kingston. ${ }^{1}$ Memorial University, Canada

10.1136/OEM-2021-EPI.332

Introduction There is a limited understanding of what is known about the implications of recent occupational health and safety (OHS) protection measures on small business management and employees during the coronavirus disease (COVID-19) pandemic. The study examines the different COVID-19 measures that have been used by small businesses in Miramichi, New Brunswick.

Objectives The study identifies the most common OHS protection measures in use within small businesses during COVID19 and explores whether differences exist in perspectives of employees and managers of small businesses on the most effective OHS protection measures used.

Methods Recruitment was collected through convenience sampling between February 6th, 2021 and March 9th, 2021. Participants for the online survey included business management personnel and employees from Miramichi, NB. The cross-sectional study used a web-based survey containing 25 items concerning demographics $(\mathrm{n}=7)$, experiences working during COVID-19 ( $n=7)$, and information and experiences with characteristics of personal protective equipment (PPE) used $(\mathrm{n}=11)$.

Results Results showed moderate ratings of positive endorsement (60\%) from both employers and employees on the use of COVID-19 OHS protection measures. No significant differences were found between employer and employee perceptions on the effectiveness of employed protection measures. The most frequently used protection measures utilized in these small businesses constitute the three lowest levels of control represented on the NIOSH Hierarchy of Controls: engineering controls, administrative controls, and PPE.

Conclusion This study provides new knowledge through the collection of stakeholder perspectives about how current workplace strategies to prevent the spread of COVID-19 in small businesses and may help guide future recommendations for small businesses dealing with other OHS and public health crises. BRAZIL IN 2019

${ }^{1}$ Renata Barbosa Vilaça Marques de Carvalho, Luciane Gabriele Pereira Gomes Lopes, Simone Bezerra da Silva, Ivonice Meire do Carmo Gentil, Daiane Oliveira dos Santos, Lorena Gomes Santos, Rafaela Cordeiro Freire. 'UFBA, Brazil

\subsection{6/OEM-2021-EPI.333}

Introduction Civil construction is responsible for most occupational accidents, generating social and economic losses and causing serious damage to workers' health.

Objective To characterize the cases of serious work accidents (SWA) reported in civil construction in Brazil.

Methodology: This is a descriptive study with a quantitative approach with secondary data in the public domain extracted from SWA records from Notifiable Diseases Information System occurred in 2019. Population data were obtained from the Continuous National Household Sample Survey, from the Brazilian Institute of Geography and Statistics. The incidence coefficient was calculated and presented by 100 thousand workers.

Results 10,551 cases of SWA were reported in civil construction in 2019, most of them concentrated in the Southeast (33.14\%) and South (22.72\%) regions of the country. The occupations with the highest number of registered cases were bricklayers $(70.10 \%)$, installation electricians $(12.10 \%)$ and carpenters (10.56\%). Most of the injured workers were male (99.02\%), black (57.35\%), aged between 30-59 years (73.19\%) and with incomplete elementary education (31.75\%). Accidents were mainly caused by contact with other and unspecified machinery (8.81\%), fall on and from scaffolding $(6.81 \%)$ and fall from, out of or through building or structure $(5,94 \%)$. The most affected part of the body was the hands $(27.28 \%)$ and most cases evolved with some type of disability (61.73\%). Fatal accidents stand for $3.07 \%$. The incidence coefficient was 154.64 cases/100 thousand workers.

Conclusion SWA were concentrated in the most productive regions of the country and occur mainly in adult workers, blacks and with low education. In addition, it assumes that the causes of accidents were mainly related to the work environment and the use of individual and collective protective equipment. Thus, measures for the prevention and promotion of workers' health are recommended to minimize the occurrence of work accidents.

\section{P-451 BIOLOGICAL AGE AS AN INDICATOR OF THE HEALTH STATE OF MINERS}

${ }^{1}$ Nurbek Yerdessov, Asset Izdenov. 'Karaganda Medical University, Kazakhstan

\subsection{6/OEM-2021-EPI.334}

Introduction The study of the biological age of miners living and working in the central Kazakhstan allows us to talk about such a phenomenon as premature aging. The main group of men with a moderate acceleration in the rate of aging was made up of workers in occupations with the most harmful working conditions, i.e., tunnellers and drillers with more than 10-15 years of work experience.

Objectives The purpose of our research was to study the health status of workers in the mining industry of central Kazakhstan, based on the results of determining the biological age. 
Methods To determine the biological age, the method of V.P. Voitenko, in which the indicator of biological age is compared with the value of the proper biological age, calculated by the formula using the calendar age of the subject. The number of respondents was 300 workers of mines of the following professions: 80 tunnellers, 50 drillers, 50 electrical fitters, 100 miners and 20 foremen.

Results The average biological age of workers, equal to 46.6 years, exceeded the proper biological age by 6 years (with an average work experience of 14.2 years) and was more than 10 years higher than the calendar age. This means that the aging of miners was more pronounced in comparison with the population standard of aging. It should be noted that the differences between the values of the calendar age and the proper biological age in miners increased with an increase in the calendar growth, which indicates that the rate of aging in miners was most pronounced in middle and older age.

Conclusion The average biological age of miners, equal to 45.6 years, exceeded the proper biological age by 5 years (with an average work experience of 14.5 years) and was 9 years higher than their calendar age.

\section{P-454 LOW BACK PAIN IN THE CARE SETTING: STUDY AMONG 300 HEALTHCARE STAFF FROM TWO UNIVERSITY HOSPITAL IN TUNISIA}

${ }^{1}$ Kamoun Salma, Olfa Jlassi, Lamia Bouzgarrou, Neila Chaari, Adel Amri, Irtyah Merchaoui. ${ }^{1}$ Centre Militaire de Médecine du Travail et de Sécurité Professionnelle, Tunisia

\subsection{6/OEM-2021-EPI.335}

Introduction Low back pain (LBP) is one of the leading musculoskeletal disorders and it is a disabling occupational hazard. It is also a common cause of morbidity among the healthcare staff within were more vulnerable to LBP.

Objectives This study aimed to assess prevalence of LBP among healthcare staff and identify the socio-occupational hazards of this morbidity.

Methods This is a cross-sectional study, carried out over 14 months, with 300 healthcare staff from two university hospitals in Tunisian center. It is based on an analysis of sociooccupational profile, the assesement of the Work Ability Index (WAI), the Nordic musculoskeletal questionnaire as well as assessment tests of physical condition and flexibility of the spine (hand-floor distance; sit-stand test; balance test)

Results The sex ratio of the sample was 1.06, the average age was $42.64 \pm 11.65 .20 \%$ of the healthcare staff in the sample had a BMI larger than 30 and $51.9 \%$ of them did not practice any regular sports activity. The perceived physical workload was considered " heavy " by $41.6 \%$ of the staff questioned. $75 \%$ of the healthcare staff in the sample had a good to excellent work ability according to the WAI. Over the 12 months preceding the survey, two third of the healthcare staff in the sample complained of LBP, with pain assessed as 'severe' or 'extremely severe' in $54.4 \%$ of cases. The LBP was statistically correlated with female gender $(p=0.01)$ and with impaired work ability $\left(p<10^{-3}\right)$. The average finger-ground distance was $7.10 \pm 7.5 \mathrm{~cm}$ and it was statistically correlated with LBP $(\mathrm{p}=0.05)$, with age $\left(\mathrm{p}<10^{-3}\right)$, with seniority $\left(\mathrm{p}<10^{-3}\right)$ and with a BMI $\geq 25$ $\left(\mathrm{p}=10^{-3}\right)$.

Conclusion LBP persists as a real health problem among healthcare staff with heavy professional and social repercussions. Reducing their prevalence and impact is based on improving working conditions and multidisciplinary care.

\section{P-455 ASSESSING QUALITY OF LIFE, PRODUCTIVITY LOSS AND ACTIVITY IMPAIRMENT IN ASTHMA}

${ }^{1}$ Amira Omrane, Olfa Jlassi, Raja Romdhani, Nadia Boudaouara, Imen Touil, Soumaya Bouchareb, Lamia Bouzgarrou, Taoufik Khalfallah, Leila Boussoffara, Jalel Knani. ${ }^{1}$ Faculty of Medicine of Monastir, Tunisia

\subsection{6/OEM-2021-EPI.336}

Background Asthma can be associated with substantial quality of life (QoL) alteration, productivity loss and activity impairment. We sought to assess the quality of life and the performance characteristics of an asthma-specific adaptation of the Work Productivity and Activity Impairment Questionnaire (WPAI:Asthma) in Tunisian young and active patients with asthma

Patients and Methods A prospective study was carried out during a year in active asthmatic patients, aged less than 65 years and consulting in the pulmonology department of a public hospital in central region of Tunisia. The WPAI:Asthma was administered to assess productivity loss. Asthma Quality-of-Life Questionnaire (AQLQ) in its validated arabic version was used to assess the QoL.

Results One hundred and one patients were enrolled in this study. Patients had a mean age of $44.2 \pm 13.2$ years and were predominantly female (66.3\%). The mean job tenure was 7.3 \pm 7.7 years. Workers belonged to the textile sector in $29.3 \%$ of cases. Asthma was allergic in $84.2 \%$ of cases and occupational in 18.2\%. Asthma was uncontrolled in $62.4 \%$ and worsened at work in $71.3 \%$. The mean AQLQ score was $5.08 \pm 1.2$. This score was statistically correlated with the occupational etiology $(p=0.001)$, the worsening of asthma symptoms at work $(p=0.016)$, the absence of professional reclassification $(p=0.002)$, the severity asthmatic disease $(p=$ $0.001)$ and uncontrolled asthma $(p=0.001)$. Absenteeism was $4.2 \pm 14.6 \%$ while presenteeism was $33.1 \pm 25.9 \%$. The resulting loss of productivity was $30.4 \pm 22.2 \%$. Activity impairment was statistically correlated with female gender $(p=0.02)$, alcohol consumption $(p=0.001)$, occupational etiology $(p=0.03)$ and uncontrolled asthma $(\mathrm{p}=0.001)$.

Conclusion This study revealed significant levels of activity impairment and QoL alteration among active and young patients suffering from asthma. Improving asthma control, working condition and mental health may be important targets for enhancing workplace productivity in asthma. Presenteeism, absenteeism and productivity loss may represent key metrics to assess intervention effectiveness in active and young patients.

\section{P-457 WORK ORGANIZATION AS A PREVENTIVE FACTOR OF WORK-RELATED LOW BACK PAIN IN TUNISIAN CRAFTSMEN}

${ }^{1}$ Asma Kheder, Ben Afia Latifa, Meriem Heni, Amira Omrane, Olfa Jlassi, Nesrine Mars, Harrathi Chayma, Taoufik Khalfallah, Lamia Bouzgarrou. 'University of Monastir - School of Medicine, Tunisia

\subsection{6/OEM-2021-EPI.337}

Introduction Work-related low back pain (WRLBP) is a complex phenomenon accounting for a high proportion of 